NBSIR 78-1478

DO NOT ED?

\title{
A Universal Set of Test Data for Computer Implementations of Elementary Mathematical Functions
}

Daniel W. Lozier

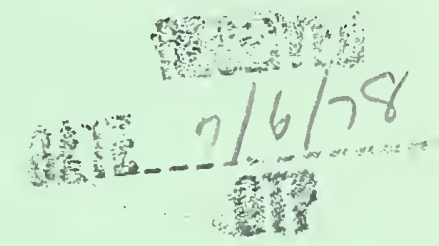

Mathematical Analysis Division

Center for Applied Mathematics

National Engineering Laboratory

National Bureau of Standards

Washington, D.C. 20234

Final Report

May 1978

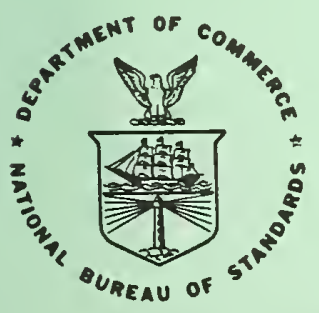

U.S. DEPARTMENT OF COMMERCE

NATIONAL BUREAU OF STANDARDS 



\section{A UNIVERSAL SET OF TEST DATA FOR COMPUTER IMPLEMENTATIONS OF ELEMENTARY MATHEMATICAL FUNCTIONS}

Daniel W. Lozier

Mathematical Analysis Division Center for Applied Mathematics National Engineering Laboratory National Bureau of Standards

Washington, D.C. 20234

Final Report

May 1978

U.S. DEPARTMENT OF COMMERCE, Juanita M. Kreps, Secretary Dr. Sidney Harman, Under Secretary Jordan J. Baruch. Assistant Secretary for Science and Technology NATIONAL BUREAU OF STANDARDS. Ernest Ambler, Director 



\section{Introduction}

Computer implementations of algorithms for the evaluation of mathematical functions have been an essential feature of computer systems since the very first days of the stored-program corputer. This does not mean, however, that these implementatlons have always been done correctly. Because of the fundamental importance of accurate function evaluation, the programer needs to have the utmost confidence in the quality of computed function values. Comparing computed function values with published tables in an effort to gain this confidence is often unsatisfactory because either the precision or argument range of the tabulated function is inadequate. Published tables were often intended as an aid to hand computation. The density of data in such tables is highest in regions of presumed highest usage, whereas most possible inputs to a computer subroutine lie outside these regions. Whus trare is a need for test data that is specifically created for the purpose of testing computer subroutines.

The ideal approach would be to create a different set of test data for each particular computer implementation of a function. Then every feature of the algorithm, the computer arithmetic, or the actual coded implementation could be tested. But this ideal would require a means of generating function values correct to the precision of the subroutine being tested. The tester may lack 
such a capability or the will to use it. The approach taken here is much more modest. The objective is to provide a small amount of data chosen to sample the function over a large range of arguments and in regions where special difficulties might be expected. The serious limitations of such an approach will become apparent later in this note.

The data presented here was developed about four years ago in response to needs at the NBS. No general method of data selection was used, and other data could undoubtedly be found that would serve the purpose as well or better. More recently, a project has been started to develop a general theory of test data selection for mathematical functions that we hope will lead to a sound and unified basis for mathematical function testing in the future.

\section{Interpreting Test Results}

The reader will note that the test data is presented in decimal form with five digit test arguments. Although many pocket calculators use decimal arithmetic, most computers use binary (or hexadecimal.). The data may be used to test decimal or non-decimal implementations of functions, but the interpretation of the results is slightly different in these two cases. Let us first consider the decimal case. We may as well assume the computing device is a calculator. In this case 
there is no conversion between incommensurate internal and external number bases. The test arguments are exactly representable, provided at least five digits can be accommodated by the calculator, so any error in the function value is entirely attributable to the implementation.

This section is mainly concerned with real functions of one real variable, although test data is given in this report for other functions. The other functions have simple definitions in terms of the real functions for which test data is presented. It is assumed that computer implementations will make use of this fact. Therefore, data for the other functions is intended only to test these definitions.

The calculator very likely uses one or more guard figures. These are extra decimal places that are used for calculations but are not ordinarily displayed. Their purpose is to rake all of the displayed figures correct for most calculations.

Let us consider the effect of guard digits on the trigonometric functions. These functions make the heaviest demand for guard figures, of the functions considered in this note. Let $t(x)$ be one of the trigonometric functions, and let $p$ be the period of $t(x)$. We first consider $x$ restricted to the interval

$$
0<\mathrm{x} \leq \mathrm{P} \text {. }
$$

Then, depending on the particular trigonometric function, $t(x)$ has 0,1 , or 2 zeros and 0,1 , or 2 poles in this interval. 
(Note that we are excluding the left end point.) In a neighborhood of a zero $x_{0}$ or a pole $x_{\infty}, t(x)$ is asymptotic to $\pm\left(x-x_{0}\right)$ or $\pm\left(x-x_{\infty}\right)^{-1}$. Let $x^{*}$ denote either a zero or a pole. In order to compute $t(x)$ in a neighborhood of $x *$, the difference $\mathrm{x}-\mathrm{x}$ * must be formed. Suppose the normally displayed precision of the calculator is $\mathrm{d}$ digits, and suppose $\mathrm{x}$ is exactly representable. As $\mathrm{x}$ approaches $\mathrm{x}$, there is an increase in the number of leading digits that are the same. Therefore, there is a progressive loss of precision through cancellation that occurs in forming $\mathrm{x}-\mathrm{x}^{*}$, as $\mathrm{x}$ gets closer to $\mathrm{x} *$. Guard digits mitigate this loss; let us see how.

Suppose $g$ guard digits are used, and that the period $p$ is known to $d+g$ digits. Then $x^{*}$ is known essentially to $d+g$ digits also, because the zeros and poles of the trigonometric function are rational fractions of $p$. Since, for $x$ in radians, $x^{\text {th }}$ is irrational, its $(d+1)$ st digit is unlikely to be zero. Thus not more than $d$ digits are likely to match between $\mathrm{x}$ and $\mathrm{x}$. Put another way, at least $g$ digits will usually survive in the difference $\mathrm{x}-\mathrm{x}^{*}$. This suggests that nothing much is to be gained by taking more than d guard digits.

We remark that $g=d$ permits the calculation of $t(x)$ to full relative precision even in the neighborhoods of zeros and poles. This advantage is illusory if $x$ is not stored exactly, as will be the usual case when $x$ is generated by prior calculation. In this case the digits of $x$ would not be known beyond 
the $d-t h$, and there would be no basis for forming $x-x^{*}$ beyond the d-th digit. It is common, therefore, to advocate the use of no guard digits ${ }^{\dagger}$ in neighborhoods of zeros of functions. This corresponds to using the absolute error criterion rather than the relative error criterion in the calculation of $t(x)$.

Because only one irrational number is needed to specify all zeros and poles of the trigonometric functions, and because the other elementary functions commonly found in computer libraries require not more than d guard digits, an unoificial standard is adhered to by many of today's digital computers: function values are computed to full relative accuracy through the use of up to d guard digits, as necessary. In calculators, however, we typically find $g<d$. Thus in calculators, if $x^{*}$ is approached too closely, then $t\left(x^{*}\right)$ will have reduced relative precision. The better calculators display only the digits that are likely to be correct.

So far we have considered $t(x)$ only for $x$ in a single period interval. Now suppose $x$ satisfies

$$
n p<x \leq n p+p
$$

for some integer $n$. Then the usual way of proceeding is to determine the number $\mathrm{pf}$ such that $0<f \leq 1$ and

$$
x=p n+p f .
$$

+ At least one guard digit is essential to obtain desirable rounding error behavior in floating-point arithmetic. See olver [4] or Sterbenz [5] for a full account. 
Then the relation

$$
t(x)=t(p f)
$$

may be employed. Determination of pf is done, in effect, by dividing $x$ by $p$, taking the integer part of the result, subtracting this from $x / p$ and finally multiplying by $p$. The subtraction step causes a loss of precision in $f$, which will be propagated to pf. Clearly, a measure of the number of digits lost is the number of digits in the integer part of $x / p$, which is given by

$$
\text { int }\left\{\log _{10}\left|\frac{x}{p}\right|\right\} \text {. }
$$

Here, int $\{\cdot\}$ is the integer part of the quantity in braces. If $g$ guard digits are used, then the number of significant digits $s$ in $p f$ is estimated by

$$
s=d+g-\operatorname{int}\left\{\log _{10}\left|\frac{x}{p}\right|\right\} .
$$

If $g=d$, then $s \geq d$ as long as $x$ satisfies $x \leq 10^{d} p$. Recalling our earlier remarks, we see that if $d$ guard digits are used, then full relative precision can be maintained uniformly, not only for $x$ in the fundamental period interval, but for very large values of $x$ as well. A new feature of the present analysis is that a fixed number of guard digits does not suffice for all $\mathrm{x}$; computer implementations of $t(x)$ must have an error path for inputs that are too large. We remark, as we did before, that $g=d$ can in no way be considered an irrefutable requirement. The advantage, as before, is only of value for exactly representable arguments. 
Before turning to a consideration of non-decimal implementations, let us briefly consider how to interpret the results of tests based on comparing the computed function values against the data. It is clear that successful comparison to the full displayed precision of the calculator does not guarantee the function is accurate at other arguments. An attempt has been made, however, to select the data so that this would be a good indication that the accuracy of the function is nearly constant throughout the tested range. Obviously, the loophole here is that the data cannot test the algorithmic details of the computer implementation. There may be an interval of arguments, perhaps very short but with reasonable probability of use under normal circumstances, for which an approximation to the function is incorrectly coded or improperly chosen. It is highly unlikely that such an interval would be tested by any universal set of test data.

On the other hand, if some of the computed function values do not compare well against the data, it does not necessarily mean the function is unacceptable. The preceding discussion of trigonometric functions indicates there are limits to what one can reasonably require. It is reasonable for a subroutine designer to specify a function so that loss of accuracy occurs under certain stated conditions. One purpose of testing is to verify that a subroutine conforms to the specification of its designer. The tester, however, may not know what specification the designer had in mind. But he can infer quite a lot about the function from 
the test results, such as whether the internal base of calculation is two or ten (as we shall see) and what the number of guard figures is. In this way he can construct a plausible model of the algorithm that will explain the test results. Without such a model, he has no basis for predicting the accuracy of the function at other arguments.

Here again we see how the power of testing with a universal set of data is limited. Without the possibility of generating additional test data, there is no way the tester can refine his model of the algorithm.

When the implementation is non-decimal, the test arguments are not necessarily exactly representable in the computer. Thus the subroutine may receive not $x$, but an approximation $\widetilde{x}$. Let $\beta$ be the base and $d$ the number of base- $\beta$ digits in the computer arithmetic. Then it is known that, with proper base conversion procedures, the relative error $\varepsilon$ in $\widetilde{x}$ satisfies a bound at least as good as

$$
|\varepsilon| \leq \beta^{1-d}
$$

Thus the error in representing $x$, if indeed the error is non-zero, is dependent only on parameters of the floatingpoint representation. The error in $f(x)$ resulting from an error in $\mathrm{x}$, however, satisfies no bound independent of $\mathrm{x}$ 
and $f$. The size of the error in $f(x)$ is predicted by standard analytical (calculus) methods and a catalog of cases will not be given here. Three handbooks of particular value are given in the references $[1,2,3]$.

By what we have just stated, the test data is divided into two classes: that for which $x$ is exactly representable, and that for which this is not true. Interpretation of test results for data of the flrst class is identical to what we stated for the case of decimal implementations. For data of the second class, the tester must take into account the effect that an input error of the indicated size will have on the function value. Any loss of accuracy that is predicted by this effect is intrinsic and cannot be reduced by any such device as the use of grard digits. Thus the tester may observe dramatic differences in the sizes of the errors in function values at neighboring arguments, just because one argument is exactly representable and another is not.

\section{The Test Data}

The functions for which test data is given are listed in Table 1. There are 13 real functions, five complex functions, the complex modulus, and the special arctangent function of two variables that is useful for converting from rectangular coordinates to polar coordinates. In the table, $x$ is a real variable and $z$ is a complex variable. All angles are expressed in radians. 
Table 1. Functions for which test data is given.

$\begin{array}{lll}e^{x} & \sin x & e^{z} \\ \sinh x & \cos x & \ln z \\ \cosh x & \tan x & \sin z \\ \tanh x & \arcsin x & \cos z \\ \sqrt{ } x & \arccos x & |z| \\ \ln x & \arctan x & \arctan \left(x_{1}, x_{2}\right) \\ \log _{10} x & & \end{array}$

The test data is presented in Tables 2, 3 and 4. There is one sample point per line in each table, and each line has 80 positions. A function identification code and seauence number appear in positions 74-80 of each line. Table 2 presents the data for real functions $g(x)$. The line format is

\begin{tabular}{l} 
Starting \\
Position \\
\hline
\end{tabular}

1

12

25

42

\section{Width}

10

12

16

21
Content

$x, 5$ D

$g(x), 7 D$

$g(x), 11 D$

$g(x), 16 D$ 
Table 3 presents the data for complex functions $g(z)$. The line format is

\section{Starting \\ Position}

1

12

23

36

Width

10

10

12

12
Content

$\operatorname{Re}(z), 5 D$

$\operatorname{Im}(z), 5 \mathrm{D}$

$\operatorname{Re}\{g(z)\}, 7 D$

$\operatorname{Im}\{g(z)\}, 7 D$

Table 4 presents the data for $g\left(x_{1}, x_{2}\right)=\left|x_{1}+i x_{2}\right|$ and $g\left(x_{1}, x_{2}\right)=\arctan \left(x_{1}, x_{2}\right)$. The line format is

\section{Starting \\ Position}

1

12

23

36

53
Width

10

10

12

16

21

\section{Content}

$\mathrm{x}_{1}, 5 \mathrm{D}$

$\mathrm{x}_{2}, 5 \mathrm{D}$

$\mathrm{g}\left(\mathrm{x}_{1}, \mathrm{x}_{2}\right), 7 \mathrm{D}$

$g\left(x_{1}, x_{2}\right), 11 D$

$g\left(\mathrm{x}_{1}, \mathrm{x}_{2}\right), 16 \mathrm{D}$

The notation 5D, 7D, etc. indicates the number of decimal places to which a number is given. All function values are believed to be in error by not more than one unit in the last place. 
$.1645811431082274-37$ $.1000478369173656-34$ .1928749847963918-21 .1000361553249357-15 $.9996807949091546-08$ .1125351747192591-06 $.1000040372791142-03$ .3354626279025118-03 $.9999701864325246-02$ .1831563888873418-01 $.9090866116450398-01$ $.1353352832366127+00$ $.2000075826305572+00$ $.3333374295812052+00$ $.7165336990154719+00$ $.1000000000000000+01$ $.1284025416687741+01$ $.1648721270700128+01$ $.2000005638888059+01$ $.2718281828459045+01$ $.4000022555584032+01$ $.7999667673464062+01$ $.1000014907117064+02$ $.1600018044518101+02$ $.1484131591025766+03$ $.1000044722017863+04$ $.5987414171519782+05$ $1000074537807576+06$ $7200489933738587+11$ $.9995640721096829+11$ $.1000298184567269+21$ $.3733241996799002+33$ $.8223012714622913+37$ $-.4111506357311457+37$ $-.2592352764293536+22$ $-.4443055260253880+07$ $-.1490478825789550+04$ -.2728991719712775+02 -.3626860407847019+01 $-.8881059821876230+00$ $-.4261563523008389+00$ $-.3045202934471426+00$ $-.2002242491609640+00$ $-.1001667500198440+00$ -.1000000166666675-02 -. $1000000000016667-04$ $-.1000000000000000-10$ -.1000000000000000-24 .0000000000000000 $.1000000000000000-34$ $.1000000000000000-15$ $.1000000000000000-07$ $.1000000001666667-03$ $.1000016666750000-01$ $.9865031219861936-01$
Ident.

\section{EXP}

EXP

EXP

EXIP

EXP

EXP

EXP

EXP

EXP

EXP

EXP

EXP

EXP

EXP

EXP

EXIP

EXP

EXP

EXP

EXF

EXP

EXP

EXP

EXP

EXP

EXP

EXP

EXP

EXP

EXP

EXP

EXP

EXP

SINH

SINH

SINH

SINH

SINH

SINH

SINH

SINH

SINH

SINH 10

SINH 11

SINH 12

SINH 13

SINH 14

SINH 15

SINH 16

SINH 17

SINH 18

SINH 19

SINH 20

SINH 21

SINH 22

Table 2. Test data for the real functions. 
$g(x)$

Ident.

$.20000+00$

$.26795+00$

$.50000+00$

10000+01

$.30000+01$

$.50000+01$

$.11000+02$

$.25000+02$

$.75000+02$

$-.85000+02$

$-.50000+02$

$-.16000+02$

$-.80000+01$

$-.40000+01$

$-.20000+01$

$-.80000+00$

$-.41421+00$

$-.30000+00$

-. 19891+00

$-.10000+00$

$-.10000-02$

$-.10000-04$

-. 10000-10

$-.10000-24$ .00000

10000-34 10000-15 10000-07 10000-03 $.10000-01$ $.98491-01$ .20000+00 $.26795+00$ $.50000+00$ $10000+01$ $.30000+01$ $.50000+01$

$.11000+02$ $.25000+02$ $.75000+02$
$.2013360+00$

$.5210953+00$

$.1175201+01$

$.1001787+02$

$.7420321+02$

$.2993707+05$

$.3600245+11$

$.1866621+33$

$.4111506+37$

$.2592353+22$

$.4443055+07$

$1490479+04$

$.2730823+02$

$.3762196+01$

$.1337435+01$

$.1087019+01$

$.1045339+01$

$.1019848+01$

$.1005004+01$

.1000001+01

1000000+01

$.1000000+01$

$.1000000+01$

$.1000000+01$

$.1000000+01$

$.1000000+01$

$.1000050+01$

$.1004854+01$

$.1020067+01$

$.1036114+01$

$.1127626+01$

$.1543081+01$

$.1006766+02$

$7420995+02$

$.2993707+05$

$.3600245+11$

$1866621+33$
$.2711679+00$

$.1000000+01$

$.1000000+01$
$.20133600254+00$

$.27116787342+00$

$.52109530549+00$

$.11752011936+01$

$.10017874927+02$

$.74203210578+02$

$.29937070849+05$

$.36002449669+11$

$.18666209984+33$

$.41115063573+37$

$.25923527643+22$

$.44430552603+07$

$.14904791613+04$

$.27308232836+02$

$.37621956911+01$

$.13374349463+01$

$.10870185079+01$

$.10198479053+01$

$.10050041681+01$

$.10000000000+01$

$.10000000000+01$

$.10000000000+01$

$.10000000000+01$

$.10000000000+01$

$.10000000000+01$

$.10000000000+01$

$.10000000050+01$

$.10000500004+01$

$.10048541606+01$

$.10200667556+01$

.10361139009+01

$.11276259652+01$

$.15430806348+01$

$.10067661996+02$

$.74209948525+02$

$.29937070866+05$

$.36002449669+11$

$.18666209984+33$
$.10453385141+01$

$.10000005000+01$

$.2013360025410940+00$

$.2711678734220111+00$

$.5210953054937474+00$

$.1175201193643801+01$

$.1001787492740990+02$

$.7420321057778876+02$

$.2993707084924806+05$

$.3600244966869294+11$

$.1866620998399501+33$

$.4111506357311457+37$

$.2592352764293536+22$

$.4443055260253993+07$

$.1490479161252178+04$

$.2730823283601649+02$

$.3762195691083631+01$

$.1337434946304845+01$

$.1087018507941036+01$

$.1045338514128860+01$

$.1019847905303566+01$

$.1005004168055804+01$

$.1000000500000042+01$

$.1000000000050000+01$

$.1000000000000000+01$

$.1000000000000000+01$

$.1000000000000000+01$

$.1000000000000000+01$

$.1000000000000000+01$

$.1000000000000000+01$

$.1000000005000000+01$

$.1000050000416668+01$

$.1004854160610825+01$

$.1020066755619076+01$

$.1036113900870081+01$

$.1127625965206381+01$

$.1543080634815244+01$

$.1006766199577777+02$

$.7420994852478784+02$

$.2993707086594976+05$

$.3600244966869294+11$

$.1866620998399501+33$

$. .1000000000000000+01$

$-.10000+26-.1000000+01$

$-.10000+03-.1000000+01$

$-.10000000000+01$

$-.10000000000+01$

$-.1000000000000000+01$

$-.85000+02$

$-.50000+02$

$-.16000+02$

$-.1000000+01$

$-.10000000000+01$

$-.1000000000000000+01$

$-.1000000000000000+01$

$-.9999999999999747+00$

$-.80000+01$

$-.1000000+01$

$-.10000000000+01$

$-.10000000000+01$

$-.9999997749296759+00$

$-.40000+01$

$-.9999998+00$

$-.99999977493+00$

$-.9993292997390670+00$

$-.20000+01-.9640276+00$

$-.99932929974+00$

$-.9640275800758169+00$

$-.80000+00-.6640368+00$

$-.96402758008+00$

$0-.6640367702678490+00$

$-.41421+00-.3920415+00$

$-.66403677027+00$

$-.3920414870470219+00$

$-.30000+00-.2913126+00$

$-.39204148705+00$

$-.2913126124515909+00$

$-.19891+00-.1963276+00$

$-.29131261245+00$

$-.1963275583738789+00$

$-.9966799462495582-01$

$-.10000+00-.9966799-01$

-.99667994625-01

$-.99999966666668000-03$

-.10000-02 -.9999997-03

$-.99999966667-03$

$-.99999999996666667-05$
SINH 23

SINH 24

SINHI 25

SINHI 26

SINH 27

SINH 28

SINH 29

SINH 30

SINH 31

$\mathrm{COSH} 1$

$\mathrm{COSH} 2$

$\mathrm{COSH} 3$

$\mathrm{COSH} 4$

COSH 5

$\mathrm{COSH} \quad 6$

$\mathrm{COSH} 7$

$\mathrm{COSH} 8$

$\mathrm{COSH} 9$

$\mathrm{COSH} 10$

$\mathrm{COSH} 11$

$\mathrm{COSH} 12$

$\mathrm{COSH} 13$

COSH 14

COSH 15

$\mathrm{COSH} 16$

$\mathrm{COSH} 17$

COSH 18

COSH 19

$\mathrm{COSH} 20$

$\mathrm{COSH} 21$

$\mathrm{COSH} 22$

$\mathrm{COSH} 23$

$\mathrm{COSH} 24$

COSH 25

COSH 26

$\mathrm{COSH} 27$

$\mathrm{COSH} 28$

$\mathrm{COSH} 29$

COSH 30

$\mathrm{COSH} 31$

TANH 1

TANH 2

TANH 3

TANH 4

TANH 5

TANH 6

TANH 7

TANH 8

TANH 9

TANH 10

TANH 11

TANH 12

TANH 13

TANH 14

TANH 15

Table 2 (cont.). Test data for the real functions. 

-. 10000-10

$-.10000-24$ .00000

$-.1000000-10$ .0000000

$.10000-34$

$.10000-15$

$.10000-07$

10000-03

$.10000-01$

$.98491-01$

$.20000+00$

$.26795+00$

$.50000+00$

$.10000+01$

$.30000+01$

$.50000+01$

$11000+02$

$.25000+02$

$.75000+02$

$.10000+11$

$.10000+36$

.00000

$.10000-34$

$.10000-15$

$.10000-07$

10000-03

$.10000-01$

$.98491-01$

.20000+00

$26795+00$

$.50000+00$

$.10000+01$

$.30000+01$

$.50000+01$

$.11000+02$

$.25000+02$

$.75000+02$

$.10000+11$

$.10000+36$

$.10000-19-.4605170+02$

$.10000-07-.1842068+02$

$.11254-06-.1599996+02$

$.10000-04$

$-.1151293+02$

$.33546-03$

$-.8000008+01$

$.10000-01$

$-.4605170+01$

$.18316-01$

$-.3999980+01$

$.31250-01$

$-.3465736+01$

$.90909-01$

$-.2397896+01$

$.13534+00$

$-.1999965+01$

$.20000+00$

$-.1609438+01$

$.33333+00$

$-.1098622+01$

$-.6931472+00$

$50000+00$

$-.2876821+00$

$-.1053605+00$

-.1005034-01

$90000+0$

$.99000+00$

$-.1000500-02$
$-.10000000000-10$

$-.10000000000-24$

.00000000000

$.10000000000-34$

$.10000000000-15$

$.10000000000-07$

$.99999999667-04$

$.99996666800-02$

98173760995-01

$.19737532022+00$

$.26171627771+00$

$.46211715726+00$

$.76159415596+00$

$.99505475369+00$

$.99990920426+00$

$.99999999944+00$

$.10000000000+01$

$.10000000000+01$

$.10000000000+01$

$.10000000000+01$

.00000000000

.31622776602-17

$.10000000000-07$

$.10000000000-03$

$.10000000000-01$

$.10000000000+00$

$.31383275801+00$

$.44721359550+00$

$.51763887026+00$

$.70710678119+00$

$.10000000000+01$

$.17320508076+01$

$.22360679775+01$

. $33166247904+01$

$.50000000000+01$

$.86602540378+01$

$.10000000000+06$

$.31622776602+18$

$-.46051701860+02$

$-.18420680744+02$

- .15999957123+02

$-.11512925465+02$

$-.80000078337+01$

$-.46051701860+01$

$-.39999802842+01$

$-.34657359028+01$

$-.23978962728+01$

-.19999651482+01

$-.16094379124+01$

$-.10986222887+01$

$-.69314718056+00$

$-.28768207245+00$

$-.10536051566+00$

-.10050335854-01

$-.10005003336-02$
$.1000000000000000-10$

$.1000000000000000-24$

.0000000000000000

$.1000000000000000-34$

$.1000000000000000-15$

$.1000000000000000-07$

$.9999999966666667-04$

$.9999666679999460-02$

$.9817376099498100-01$

$.1973753202249040+00$

$.2617162777126113+00$

$.4621171572600098+00$

$.7615941559557649+00$

$.9950547536867305+00$

$.9999092042625951+00$

$.9999999994421064+00$

$.1000000000000000+01$

$.1000000000000000+01$

$.1000000000000000+01$

$.1000000000000000+01$

.0000000000000000

.3162277660168379-17

$.1000000000000000-07$

$.1000000000000000-03$

.1000000000000000-01

$.1000000000000000+00$

$.3138327580097400+00$

$.4472135954999579+00$

$.5176388702560889+00$

$.7071067811865475+00$

$.1000000000000000+01$

$.1732050807568877+01$

$.2236067977499790+01$

$.3316624790355400+01$

$.5000000000000000+01$

$.8660254037844386+01$

$.1000000000000000+06$

.3162277660168379+18

$-.4605170185988091+02$

$-.1842068074395237+02$

$-.1599995712294128+02$

$-.1151292546497023+02$

$-.8000007833697665+01$

$-.4605170185988091+01$

$-.3999980284187288+01$

$-.3465735902799727+01$

$-.2397896272798871+01$

$-.1999965148178058+01$

$-.1609437912434100+01$

$-.1098622288718110+01$

$-.6931471805599453+00$

$-.2876820724517809+00$

$-.1053605156578263+00$

-.1005033585350144-01

$-.1000500333583534-02$
TANH 16

TANII 17

TANF 18

TANH 19

TANH 20

TANH 21

TANH 22

TANH 23

TANH 24

TANH 25

TANH 26

TANH 27

TANH 28

TALNH 29

TANH 30

TANH 31

TANH 32

TANF 33

TANH 34

TANH 35

SQRT ?

SQRT 2

SQRT 3

SQRT 4

SQRT 5

SQRT 6

SQRT 7

SQRT 8

SQRT 9

SQRT 10

SQRT 11

SQRT 12

SQRT 13

SQRT 14

SQRT 15

SORT 16

SQRT 17

SQRT 18

IDGE 1

LOGE 2

IOGE 3

IOGE 4

IOGE 5

LOGE 6

IOGE 7

IOGE 8

IOGE, 9

IOGE 10

IOGE 11

IOGE 12

IOGE 13

IOGE 14

IOGE 15

IOGE 16

IOGE 17

Table 2 (cont.). Test data for the real functions. 

$.99990+0$

$.99999+00$

$.10000+01$

$.10001+01$

$.10010+01$

$.10100+01$

$.11000+01$

$.15000+01$

$.20000+01$

$.27183+01$

$.40000+01$

$.80000+01$

$.10000+02$

$.16000+02$

$.20086+02$

$.64000+02$

$.14841+03$

$.10000+04$

$.10000+05$

$.59874+05$

$.10000+11$

$.10000+36$

$.10000-19$

$.10000-07$

$.11254-06$

$.10000-04$

$.33546-03$

$.10000-01$

$.18316-01$

.31250-01

.90909-01

$.13534+00$

$.20000+00$

$.33333+00$

$.50000+00$

$.75000+00$

$.90000+00$

$.99000+00$

$.99900+00$

$.99990+00$

$.99999+00$

$.10000+01$

$.10001+01$

$.10010+01$

$.10100+01$

$.11000+01$

$.15000+01$

$.20000+01$

$.27183+01$

$.40000+01$

$.80000+01$

$.10000+02$

$.16000+02$

$.20086+02$

$.64000+02$
$-.1000050-03$

$-.1000005-04$

.0000000

$.9999500-04$

$.9995003-03$

.9950331-02

$.9531018-01$

$.4054651+00$

$.6931472+00$

$.1000007+01$

$.1386294+01$

$2079442+01$

$.2302585+01$

$.2772589+01$

$.3000023+01$

$.4158883+01$

$.4999979+01$

$.6907755+01$

$.9210340+01$

$.1100000+02$

$.2302585+02$

$.8059048+02$

$-.2000000+02$

$-.8000000+01$

$-.6948693+01$

$-.5000000+01$

$-.3474359+01$

$-.2000000+01$

$-.1737169+01$

$-.1505150+01$

$-.1041393+01$

$-.8685738+00$

$-.6989700+00$

$-.4771256+00$

$-.3010300+00$

$-.1249387+00$

$-.4575749-01$

-.4364805-02

$-.4345118-03$

$-.4343162-04$

$-.4342967-05$

.0000000

$.4342728-04$

$.4340775-03$

$.4321374-02$

$.4139269-01$

$.1760913+00$

$3010300+00$

$.4342974+00$

$.6020600+00$

$.9030900+00$

$.1000000+01$

$.1204120+01$

$.1302893+01$

$.1806180+01$
-. 10000500033-03

$-.1000050003333583-03$

$-.1000005000033334-04$

.00000000000

$.99995000333-04$

$.99950033308-03$

$.99503308532-02$

$.95310179804-01$

$.40546510811+00$

$.69314718056+00$

$.10000066849+01$

$.13862943611+01$

$.20794415417+01$

$.23025850930+01$

$.27725887222+01$

$.30000230550+01$

$.41588830834+01$

$.49999787139+01$

$.69077552790+01$

$.92103403720+01$

$.10999997633+02$

$.23025850930+02$

$.80590478255+02$

$-.20000000000+02$

$-.80000000000+01$

$-.69486930892+01$

$-.50000000000+01$

$-.34743592574+01$

$-.20000000000+01$

$-.17371693651+01$

$-.15051499783+01$

-.10413931195+01

$-.86857382785+00$

$-.69897000434+00$

$-.47712559769+00$

$-.30102999566+00$

$-.12493873661+00$

-.45757490561-01

$-.43648054025-02$

$-.43451177402-03$

$-.43431619808-04$

-.43429665339-05

.00000000000

$.43427276863-04$

$.43407747932-03$

.43213737826-02

41392685158-01

$.17609125906+00$

. $30102999566+00$

$.43429738512+00$

$60205999133+00$

$.90308998699+00$

$10000000000+01$

$12041199827+01$

$13028934584+01$

. $18061799740+01$
.0000000000000000

$.9999500033330834-04$

$.9995003330835332-03$

$.9950330853168083-02$

$.9531017980432486-01$

$.4054651081081644+00$

$.6931471805599453+00$

$.1000006684913988+01$

$.1386294361119891+01$

$.2079441541679836+01$

$.2302585092994046+01$

$.2772588722239781+01$

$.3000023054971147+01$

$.4158883083359672+01$

$.4999978713907727+01$

$.6907755278982137+01$

$.9210340371976183+01$

$.1099999763311237+02$

$.2302585092994046+02$

$.8059047825479160+02$

$-.2000000000000000+02$

$-.8000000000000000+01$

$-.6948693089182026+01$

$-.5000000000000000+01$

$-.3474359257357683+01$

-. $2000000000000000+01$

- .1737169365144340+01

-.1505149978319906+01

$-.1041393119452924+01$

$-.8685738278525498+00$

$-.6989700043360188+00$

$-.4771255976861963+00$

- .3010299956639812+00

-. $1249387366083000+00$

-.4575749056067513-01

-.4364805402450085-02

$-.4345117740176913-03$

$-.4343161980751038-04$

$-.4342966533901379-05$

.0000000000000000

$.4342727686266964-04$

$.4340774793186407-03$

$.4321373782642574-02$

$.4139268515822504-01$

$.1760912590556812+00$

$.3010299956639812+00$

$.4342973851245086+00$

$.6020599913279624+00$

$.9030899869919436+00$

$.1000000000000000+01$

$.1204119982655925+01$

$.1302893458356505+01$

$.1806179973983887+01$
LOGE 18

LOGE 19

IOGE 20

LOGE 21

IOGE 22

LOGE 23

IOGE 24

LOGE 25

IOGE 26

LOGE 27

LOGE 28

LOGE 29

LOGE 30

IOCE 31

IOGE 32

IOGE 33

LOGE 34

IOGE 35

LOGE 36

LOGE 37

IOGE 38

LOGE 39

IG10 1

IG10 2

LG10 3

IG10 4

IG10 5

IG10 6

IG10 7

LG10 8

IG10 9

IG10 10

LG10 11

IG10 12

LG10 13

LG10 14

LG10 15

LG10 16

LG10 17

IG10 18

LG10 19

LG10 20

LG10 21

IG10 22

LG10 23

IG10 24

IG10 25

LG10 26

IG10 27

LG10 28

LG10 29

LG10 30

LG10 31

IG10 32

LG10 33

Table 2 (cont.). Test data for the real functions. 

$\mathrm{g}(\mathrm{x})$

Ident.

$-.1000000-24$
$.14841+03$ $.10000+04$ $.10000+05$ $.59874+05$ $.10000+11$ $.10000+36$ $-.10000+05$ $-.10000+03$ $-.50000+02$

$-.16000+02$

$-.80000+01$

$-.40000+01$

$-.20000+01$

$-.50000+00$

$-.10000+00$

-. 10000-02

$-.10000-04$

$-.10000-10$

$-.10000-24$ .00000

$.10000-34$

$.10000-15$

$.10000-07$

$.10000-03$

$.10000-01$

$.25000+00$

$.26179+00$

$.52360+00$

$.75000+00$

$.78539+00$

$.10000+01$

$.10472+01$

$.13089+01$

$.15708+01$

$.18325+01$

$.20944+01$

$.23561+01$

.26180401

$.28797+01$

$.30000+01$

$31496+01-.7346490-05$

$34033+01-.2587301+00$

$.2171463+01$ $.3000000+01$ $.4000000+01$ $.4777238+01$ $.1000000+02$ $.3500000+02$ .3056144400 $.5063656+00$ $.2623749+00$ $.2879033 \div 00$

$-.9893582+00$ $.7568025+00$

$-.9092974+00$

$-.4 \% 94255+00$

$-.9983342-01$

$-.9999998-03$

$-.9000000-04$

$-.1000000-10$ .0000000

$.1000000-34$

$.1000000-15$

$.1000000-07$

$.1000000-03$

$.9999833-02$

$.2474040+00$

$.2588100+00$

$.5000011+00$

$.6816388+00$

$.7071010+00$

$.8414710+00$

$.8660266+00$

$.9659007+00$

$.1000000+01$

$.9659506+00$

$.8660230+00$

$.7071736+00$

$.4999947+00$

$.2589091+00$

$.1491200+00$

$.21714631659+01$ $.30000000000+01$ $.40000000000+01$ $.47772382730+01$ $.10000000000+02$ $.35000000000+02$ $.30561438889+00$ $.50636564119+00$ $.26237485370+00$ $.28790331667+00$ $-.98935824662+00$ $.75680249531+00$ $-.90929742683+00$ $-.47942553860+00$ $-.99833416647-01$ - .99999983333-03 $-.99999999998-05$ $-.10000000000-10$

$-.10000000000-24$ .00000000000

$.10000000000-34$

$.10000000000-15$

$.10000000000=07$

99999999833-04

$.99998333342-02$

$.24740395925+00$

$.25880997717+00$

$.50000106036+00$

$68163876002+00$

$.70790100877+00$

$.84147098481+00$

$.86602662818+00$

$.96590073209+00$

$.99999999999+00$

$.96595059462+00$

$.86602295497+00$

$.70717359269+00$

$.49999469818+00$

$.25890913181+00$

.94192000806400

. $43464902067-05$

$-.73464902067-05$

$-.25873013885 \% 00$

$-.50000742252+00$

$-.70704256096+00$

. $36652+01$

$-.5000074+00$

$-.7070426+00$

$-.86603030135+00$

$-.96590263494+00$

$41888+01$

$-.8659026+00$

$-.96590263414+00$

$.47124+01$

$.49741+0$

$-.1000000+01$

$-.99999999994+00$

$-.96594869389400$

$50000+01-.9589243400-.95892427466+00$

.52360401

$.54977+0$

$.57596+01$

$.60213+01$

$.62832+01$

$.11000+02$
.2171463165083844401 .3000000000000000401 $.4000000000000000+01$ $.4777238273009532+01$ .1000000000000000402 $.3500000000000000+02$ $.3056143888882521+00$ $.5063656411097588+00$ .2623748537039288400 $.2879033166650653 \% 00$ $-.9893582466233818+100$

$.7568024953079283+00$

$-.9092974268256817+00$

$-.4794255386042030400$

$-.9983341664682815-01$

$-.9999998333333447-03$

-.9999999999833333-05

$-.1000000000000000-10$

$-.1000000000000000-24$ .0000000000000000

$.1000000000000000-34$ $.1000000000000000-15$

$.1000000000000000-07$ .9999999983333333-04 $.9999833334966665-02$ $.24740395925452292-00$ $.2588099771734655+00$ .5000010603626028400 $.6816387600233342+00$ $.7071010087692932+00$ $.8444709848078965+00$ $.8660266281835432+00$ $.9659007320923110+00$ $.9999999999932538+00$ $.8660229549706499+00$ $.7071735926855460+00$ $.4999946981757422+00$ $.2589091318126125+00$ $.1411200080598672+00$ $-.7346410206695457-05$ $-.2587301388516791 \div 00$ $-.5000074225224785+00$ $-.7070425609630992+00$ $-.8660303013496971+00$ $-.9659026341449996+00$ $-.9999999999392840+00$ $-.9659486938865708+00$ $-.9589242746631385+00$ $-.8660192817110177+00$ $-.7071683984608595+00$ - .4999883359618970400 $-.2589020358956423+00$ $.1469282041299443=04$ $-.9999902065507035+00$ $.9659505946243037+00$

$\begin{array}{lc}\text { IGIO } & 34 \\ \text { IGIO } & 35 \\ \text { IGIO } & 36 \\ \text { IGIO } & 37 \\ \text { IGIO } & 38 \\ \text { IGI0 } & 39 \\ \text { SIN } & 1 \\ \text { SIN } & 2 \\ \text { SIN } & 3 \\ \text { SIN } & 4 \\ \text { SIN } & 5 \\ \text { SIN } & 6 \\ \text { SIN } & 7 \\ \text { SIN } & 8 \\ \text { SIN } & 9 \\ \text { SIN } & 10 \\ \text { SIN } & 11 \\ \text { SIN } & 12 \\ \text { SIN } & 13 \\ \text { SIN } & 14 \\ \text { SIN } & 15 \\ \text { SIN } & 16 \\ \text { SIN } & 17 \\ \text { SIN } & 18 \\ \text { SIN } & 19 \\ \text { SIN } & 20 \\ \text { SIN } & 21 \\ \text { SIN } & 22 \\ \text { SIN } & 23 \\ \text { SIN } & 24 \\ \text { SIN } & 25 \\ \text { SIN } & 26 \\ \text { SIN } & 27 \\ \text { SIN } & 28 \\ \text { SIN } & 29 \\ \text { SIN } & 30 \\ \text { SIN } & 31 \\ \text { SIN } & 32 \\ \text { SIN } & 33 \\ \text { SIN } & 34 \\ \text { SIN } & 35 \\ \text { SIN } & 36 \\ \text { SIN } & 37 \\ \text { SIN } & 38 \\ \text { SIN } & 39 \\ \text { SIN } & 40 \\ \text { SIN } & 49 \\ \text { SIN } & 42 \\ \text { SIN } & 43 \\ \text { SIN } & 44 \\ \text { SIN } & 45 \\ \text { SIN } & 46 \\ \text { SIN } & 47 \\ \text { SIN } & 48 \\ \text { SIN } & 49\end{array}$

Table 2 (cont.). Test data for the real functions. 

$.25000+02$

$.75000+02$

$.10000+04$

$.10000+06$

$-.10000+05$

$-.10000+03$

$-.50000+02$

$-.16000+02$

$-.80000+01$

$-.40000+01$

$-.20000+01$

$-.50000+00$

$-.10000+00$

-. 10000-02

-.10000-04

$-.10000-10$

-.10000-24

.00000

$.10000-34$

$.10000-15$

$.10000-07$

$.10000-03$

$.10000-01$

$.25000+00$

$.26179+00$

$.52360+00$

$.75000+00$

$.78539+00$

$.10000+01$

$.10472+01$

$.13089+01$

$.15708+01$

$.18325+01$

$.20944+01$

$.23561+01$

$.26180+01$

$.28797+01$

$.30000+01$

$.31416+01$

$.34033+01$

. 36652+01

$.39269+01$

$.41888+01$

$.44505+01$

$.47124+01$

$.49741+01$

$.50000+01$

$.52360+01$

$.54977+01$

$.57596+01$

$.60213+01$

$.62832+01$

$.11000+02$

$.25000+02$

$.75000+02$
$.1323518+00$
$-.3877816+00$

$.8268795+00$

.3574880-01

$-.9521554+00$

. $8623189+00$

$.9649660+00$

$-.9576595+00$

$-.1455000+00$

$-.6536436+00$

$-.4161468+00$

$.8775826+00$

$.9950042+00$

$.9999995+00$

$.1000000+01$

$.1000000+01$

$.1000000+01$

$.1000000+01$

$.1000000+01$

$.1000000+01$

$.1000000+01$

$.1000000+01$

$.9999500+00$

$.9689124+00$

$.9659283+00$

$.8660248+00$

$.7316889+00$

$.7071126+00$

$.5403023+00$

$.4999979+00$

. 2589127+00

$-.3673205-05$

$-.2587266+00$

$-.5000042+00$

$-.7070400+00$

$-.8660285+00$

$-.9659017+00$

$-.9899925+00$

$-.1000000+01$

$-.9659496+00$

$-.8660211+00$

$-.7071710+00$

$-.4999915+00$

$-.2589056+00$

. 1101962-04

$.2587337+00$

$.2836622+00$

$.5000106+00$

$.7070452+00$

$.8660321+00$

$.9659036+00$

$.1000000+01$

$.4425698-02$

$.9912028+00$

$.9217513+00$
$-.13235175010+00$ $.82687954053+00$ $.35748797972-01$

$-.3877816354094304+00$

$.8268795405320026+00$

$.3574879797201651-01$

$-.95215536826+00$

$.86231887229+00$

$.96496602849+00$

$-.95765948032+00$

$-.14550003381+0$

$-.65364362086+00$

$-.41614683655+00$

$.87758256189+00$

$.99500416528+00$

$.99999950000+00$

$.99999999995+00$

$.10000000000+01$

$.10000000000+01$

$.10000000000+01$

$.10000000000+01$

$.10000000000+01$

$.10000000000+01$

$.99999999500+00$

$.99995000042+00$

$.96891242171+00$

$.96592825599+00$

$.86602479158+00$

$.73168886887+00$

$.70711255356+00$

$.54030230587+00$

$.49999787927+00$

$.25891267977+00$

-.36732051034-05

$-.25872659072+00$

$-.50000424145+00$

$-.70703996337+00$

$-.86602846477+00$

$-.96590168313+00$

$-.98999249660+00$

$-.99999999997+00$

$-.96594964426+00$

$-.86602111835+00$

$-.70717099558+00$

$-.49999151707+00$

$-.25890558386+00$

$.11019615310-04$

$.25873368698+00$

$.28366218546+00$

$.50001060359+00$

$.70704515854+00$

$.86603213792+00$

$.96590358515+00$

$.99999999989+00$

$.44256979881-02$

$.99120281186+00$

$.92175126972+00$
$-.9521553682590149+00$

$.8623188722876839+00$

$.9649660284921133+00$

$-.9576594803233846+00$

$-.1455000338086135+00$

$-.6536436208636119+00$

$-.4161468365471424+00$

$.8775825618903727+00$

$.9950041652780258+00$

$.9999999999500000+00$

$.1000000000000000+01$

$.1000000000000000+01$

$.1000000000000000+01$

$.1000000000000000+01$

$.1000000000000000+01$

$.1000000000000000+01$

$.9999999950000000+00$

$.9999500004166653+00$

$.9689124217106448+00$

$.9659282559877158+00$

$.8660247915829389+00$

$.7316888688738209+00$

$.7071125535566795+00$

$.5403023058681397+00$

$.4999978792725456+00$

$.2589126797658577+00$

-.3673205103372509-05

$-.2587265907187627+00$

$-.5000042414459138+00$

$-.7070399633742194+00$

$-.8660284647724626+00$

$-.9659016831251715+00$

- .9899924966004455+00

- .9999999999730151+00

$-.9659496442619537+00$

$-.8660211183466762+00$

$-.7071709955779735+00$

$-.4999915170721927+00$

$-.2589055838558741+00$

$.1101961530991928-04$ $.2587336869810885+00$ $.2836621854632263+00$ $.5000106035922969+00$

$.7070451585424392+00$

$.8660321379152469+00$ $.9659035851517953+00$ $.9999999998920605+00$ $.4425697988050786-02$ $.9912028118634736+0 \mathrm{C}$ $.9217512697247493+00$
$.9999995000000417+00$
SIN 50

SIN 51

SIN 52

SIN 53

$\cos 1$

$\cos 2$

$\cos 3$

$\cos 4$

$\cos 5$

$\cos 6$

$\cos 7$

$\cos 8$

$\cos 9$

$\cos 10$

$\cos 11$

$\cos 12$

$\cos 13$

$\cos 14$

$\cos 15$

$\cos 16$

$\cos 17$

$\cos 18$

$\cos 19$

$\cos 20$

$\cos 21$

$\cos 22$

$\cos 23$

$\cos 24$

$\cos 25$

Cos 26

$\cos 27$

$\cos 28$

COS 29

Cos 30

$\cos 31$

COS 32

Cus 33

$\cos 34$

$\cos 35$

$\cos 36$

$\cos 37$

$\cos 38$

COS 39

$\cos 40$

$\cos 41$

$\cos 42$

$\cos 43$

$\cos 44$

$\cos 45$

$\cos 46$

$\cos 47$

$\cos 48$

$\cos 49$

cos 50

Cos 51

Table 2 (cont.). Test data for the real functions. 

$\mathrm{x}$

$.10000+04$

$.10000+06$

$-.10000+05$

$-.10000+03$

$-.50000+02$

$-.16000+02$

$-.80000+01$

$-.40000+01$

$-.20000+01$

$-.50000+00$

$-.10000+00$

$-.10000-02$

$-.10000-04$

$-.10000-10$

$-.10000-24$ .00000

$.10000-34$

$.10000-15$

$.10000-07$

$.10000-03$

$.10000-01$

$.25000+00$

$.26179+00$

$.52360+00$

$.75000+00$

$.78539+00$

$.10000+01$

$.10472+01$

$.13089+01$

$.15708+01$

$.18325+01$

$.20944+01$

. $23561+01$

. 26180+01

$.28797+01$

.30000+01

. 31416+01

.34033+01

. $36652+01$

.39269+01

. $41888+01$

$.44505+01$

$47924+01-.0074727+05$

$.49741+01-.3733370+01$

$.50000+01-.3380515+01$

$.52360+01-.1732002+01$

$.54977+01-.1000174+01$

$.57596+01-.5773323+00$

$.60213+01-.2680413+00$

$.62832+01 \quad .9469282=04$

$.11000+02-.2259508+03$

$.25000+02-.1335264+00$

$.75000+02-.4207010+00$

$.10000+04-.1470324+01$

$.1470324+01$
$-.3577166-01$ $\mathrm{g}(\mathrm{x})$

$.56237907629+00$

$.5623790762907030+00$

$-.99936080744+00-.9993608074382125+00$

$-.32097113462+00-.3209711346238147+00$

$.58721391516+00$

$.27190069200+00$

$5872139151569291+00$

$2799006119976307+00$

$-.30063224202+00-.3006322420239034+00$ .67997114552401

$-.91578212823+09$ $6799791455220379+01$ $.21850398633+01$

$-.54630248984+00$

$-.1157821282349578+01$ $.2185039863261519+01$

$-.5463024898437905+00$

$-.10033467209+00-.1003346720854505+00$

$-.10000003333-02-.1000000333333467-02$

-.90000000000-04-.1000000000033333-04

-. $10000000000-10-.1000000000000000-10$

$-.10000000000-24-.1000000000000000-24$

.00000000000

$.10000000000-34$

.0000000000000000

$10000000000-15$

$.10000000000-07$

$.10000000033-03$

$.10000333347-01$

$.25534192122+00$

$.26793913064+00$

$.57735190173+00$

$.93159645994+00$

$.99998367334+00$

$.15574077247+01$

$.17320606028+01$

$.37306042059+01$

$-.27224180841+06$

$-.37334801651+01$

$-.17320312173+01$

$-.10001889982+01$

$-.57734210654+00$

$-.26804915690+00$

$.1000000000000000-34$

$.1000000000000000-15$

$.1000000000000000-07$

$.1000000003333333-03$

$.1000033334666721-01$

$.2553419212210363+00$

.2679391306436292400

$.5773519017263813+00$

$.9315964599440725+00$

.9999836733383840400

$.1557407724654902+01$

$.1732060602824032+01$

$.3730604205888268+01$

$-.2722498084073549+06$

$-.3733480165068527+01$

$-.1732031217307841+01$

$-.1000188998243733+01$

$=.5773421065404694+00$

$-.2680491569027118400$

$-.1425465430742778+00$

$.7346410206893699-05$

$.73464102069-05$

$.26785054520+00$

$.57736169700+00$ $.99981838252+00$

$.17320899891+01$

$.37307137983+01$

$.2678505452003734+00$

$.5773616969953855+00$

$.2998183825189700+00$

$.1732089989088061+01$

$.3730713798288306+01$

$-.90747269466+05-.9074726946585296+05$

$-.37333704210+01$

$-.33805150062+01$

$-.3733370420981070401$

$-.3380515006246586+01$

$-.17320018325+01-.1732001832539456+01$

$-.10001743028+01-.1000174302754199+01$

$-.57733231144+00-.5773323114376475+00$

$-.25804128267+00$

$.94692820415-04$

$-.2680492826658625+00$ $.1469282049458037-04$ $-.22595084645403-.2259508464549951+03$

$-.13352640702400-.1335264070215359+00$

$-.42070095052+00$

$-.4207009506211244400$ $.14703249557+01$ .1470324155702718101
$-.3577166295289877-01$
Ident.

Cos 52

cos 53

TAN

TAN

TAN

IAN

MAN

TAN

TAN

PAN

DAN

TAN

TAN

TAN

TAN

TAN

TAN

TAN

TAN

TAN

TAN

TAN

TAN

TAN

TAN

TAN

TAN

TAN

TAN

TAN

TAN

TAN

TAN

TAN

TAN

IAN

TAN

TRN

TAN

TAN

TAN

TAN

DAN

IAN

MAN

IAN

IRN

TAN

TAN

TAN

TAN

TAN

IAN

TAN

TAN

41
1

2

3

4

5

6

7

8

9

10

12

13

94

15

16

17

18

19

20

21

22

23

24

25

26

27

28

29

30

31

32

33

34

35

36

37

38

39

40

41

42

43

44

45

46

47

48

49

50

51

52

53 

$\mathrm{x}$

$-.10000+0$

-. $99990+00$

$-.99000+00$

$-.80000+00$

$-.60000+00$

$-.40000+00$

$-.20000+00$

$-.10000-01$

$-.10000-03$

$-.10000-07$

-. 10000-15

$-.10000-34$ .00000 $.10000-24$ $.10000-10$ $.10000-04$ .10000-02 $.10000+00$ $.30000+00$ $.50000+00$ $70000+00$ $90000+00$ $.99900+00$ .99999+00 - . $10000+01$ $-.99990+00$ $-.99000+00$ $-.80000+00$ $-.60000+00$ $-.40000+00$ $-.20000+00$ $-.10000-01$ $-.10000-03$ $-.10000-07$ -. $10000-15$ $-.10000-34$ .00000 $.10000-24$ $.10000-10$ $.10000-04$ $.10000-02$ $.10000+00$ $.30000+00$ $.50000+00$ $.70000+00$ $.90000+00$ . 99900400 $.99999+00$ $-.10000+26$ $-.10000+03$

$-.85000+02$

$-.50000+02$

$-.16000+02$

$-.80000+01$

$-.40000+01$ $\mathrm{g}(\mathrm{x})$

$-.1570796+01-.15707963268+01-.1570796326794897+01$

$-.1556654+01-.15566540733+01-.1556654073317384+01$

$-.1429257+01-.14292568535+01-.1429256853470469+01$

$-.9272952+00-.92729521800+00-.9272952180016122+00$

$-.6435011+00-.64350110879+00-.6435011087932844+00$

$-.4115168+00-.41151684607+00-.4115168460674880+00$

$-.2013579+00-.20135792079+00-.2013579207903308+00$

-.1000017-01 -.10000166674-01 -.1000016667416711-01

$-.1000000-03-.10000000017-03-.1000000001666667-03$

$-.1000000-07-.10000000000-07-.1000000000000000-07$

$-.1000000-15-.10000000000-15-.1000000000000000-15$

$-.1000000-34$ .0000000

$-.10000000000-34$ .00000000000

$-.1000000000000000-34$ .0000000000000000 $.10000000000-24$ $.10000000000-10$ $.10000000000-04$ .10000001667-02 $.10016742116+00$ $.30469265402+00$ $.52359877560+00$ $.77539749661+00$ .11197695150401 $.15260712396+01$ $.15663241871+01$ .31415926536+01 . $31274504001+01$ . $30000531803+01$ $.24980915448+01$ .22142974356t01 $.19823131729+01$ $.17721542476+01$ $.15807964935+01$ $.15708963268+01$ $.15707963368+01$ $.15707963268+01$ $.15707963268+01$ $.15707963268+01$ $.15707963268+01$ $.15707963268+01$ $.15707863268+01$ $.15697963266+01$ $.14706289056+01$ $.12661036728+01$ $.10471975512+01$ $.79539883018+00$ $.45102681180+00$ $.44725087169-01$ $.44721396818-02$ $.1000000000000000-24$ $.1000000000000000-10$ $.1000000000016667-04$ $.1000000166666742-02$ $.1001674211615598+00$ . $3046926540153975 \% 00$ $.5235987755982989+00$ $.7753974966107531+00$ $.1119769514998634+01$ $.1526071239626163+01$ $.1566324187113109+01$ . $3141592653589793+01$ . $3127450400112280+01$ .3000053180265366+01 $.2498091544796509+01$ $.2214297435588181+01$ $.1982313172862385+01$ $.1772154247585227+01$ $.1580796493469064+01$ $.1570896326795063+01$ $.1570796336794897+01$ $.1570796326794897+01$ $.1570796326794897+01$ $.1570796326794897+01$ $.1570796326794897+01$ $.1570796326784897+01$ $.1570786326794896+01$ $.1569796326628230+01$ $.1470628905633337+01$ $.1266103672779499+01$ $.1047197551196598+01$ $.7953988301841436+00$ $.451026811 .7962624+00$ $.4472508716873343-01$ .4472139681787927-02 4472140-02 $-.1570796+01$ $-.15707963268+01$ $-.15607966601+01$ $-.1570796326794897+01$ $-.1560796660108231+01$ $-.1559032163645138+01$ $-.1550798992821746+01$ $-.1508377516798939+01$ $-.1446441332248135+01$ $-.1325817663668032+01$
Ident.

ASIN I

ASIN 2

ASIN 3

ASIN \&

ASIN 5

ASIN 6

ASIN 7

ASIN 8

ASIN 9

ASIN 10

ASIN II

ASIN 12

ASIN 13

ASIN 14.

ASIN 15

ASIN 16

ASIN 17

ASIN 18

ASIN 19

ASIN 20

ASIN 21

ASIN 22

ASIN 23

ASIN 24

ICOS I

ACOS 2

Acos 3

ACOS $\triangle$

Acos 5

Acas 6

Acos 7

ICOS 8

Acos 9

ACOS 10

ACOS 11

AOOS 12

ACOS 13

ACOS 14

ACOS 15

ACOS 16

ACOS 17

ACOS 18

AOOS 19

ACOS 20

Acos 21

Acos 22

ACOS 23

ACOS 24

AIAN 1

ATAN 2

ATPN 3

ATAN 4

ATAN 5

ATAN 6

ATAN 7 

$\frac{x}{-.20000+01}-. .1107149+01-.1107148717$

148717+01-.1107148717794091+01

$-.80000+00-.6747409+00-.67474094222+00-.6747409422235527+00$

$-.41421+00-.3926960+00-.39269604102+00-.3926960410192606+00$

$-.30000+00-.2914568+00-.29145679448+00-.2914567944778671+00$

$-.19891+00-.1963473+00-.19634726357+00-.1963472635716957+00$

$-.10000+00-.9966865-01-.99668652491-01-.9966865249116203-01$

-.10000-02 -.9999997-03 -.99999966667-03 -.99999996666668667-03

-.10000-04 -.1000000-04 -.99999999997-05 -.9999999999666667-05

-.10000-10-.1000000-10-.10000000000-10-.1000000000000000-10

$-.10000-24-.1000000-24-.10000000000-24-.1000000000000000-24$

.00000

$.10000-34$

$.10000-15$

$.10000-07$

$.10000-03$

$.10000-01$

$.98491-01$

$.20000+00$

$.26795+00$

$.50000+00$

$.10000+01$

$.30000+01$

$.50000+01$

$.11000+02$

$.25000+02$

$.75000+02$

$.10000+11$

$.10000+36$
.0000000

$.1000000-34$

$.1000000-15$

$.1000000-07$

$.1000000-03$

$.9999667-02$

.9817437-01

$.1973956+00$

$.2618001+00$

$.4636476+00$

$.7853982+00$

$.1249046+01$

$.1373401+01$

$.1480136+01$

$.1530818+01$

$.1557464+01$

$.1570796+01$

$.1570796+01$
.00000000000 $.10000000000-34$ $.10000000000-15$ $.10000000000-07$ $.99999999667-04$ $.99996666867-02$ $.98174370943-01$ $.19739555985+00$ $.26180014127+00$ $.46364760900+00$ $.78539816340 \div 00$ $.12490457724+02$ $.13734007669+01$ $.14801364396+01$ $.15308176397 \div 01$ $.15574637835+01$ $.15707963267+01$ $.15707963268+01$
.0000000000000000

$.1000000000000000-34$ $.1000000000000000-15$ $.1000000000000000-07$ $.9999999966666667-04$ $.9999666686665238-02$ $.9817437094269847-01$ $.1973955598498808+00$ $.2618001412710175+00$ $.4636476090008061+00$ $.7853981633974483+00$ $.1249045772398254+01$ $.1373400766945016+01$ $.1480136439594152+01$ .1530817639671607401 $.1557463783500751+01$ $.1570796326694897+01$ $.1570796326794897+01$
Ident.

AIAN 8

ATPN 9

ATAN 10

ATAN 11

ATAN 12

ATAN 13

AIVIN 14

ATAN 15

ATAN 26

ATAN 17

AMAN 18

AIAN 19

ATAN 20

ATAN 21

ATAN 22

AIAN 23

AIAN 24

AMAN 25

ATMN 26

ATAN 27

AIAN 28

AIAN 29

ATAN 30

ATAN 31

AIAN 32

ATAN 33

ATEN 34

AIPN 35

Table 2 (cont.). Test data for the real runctions. 



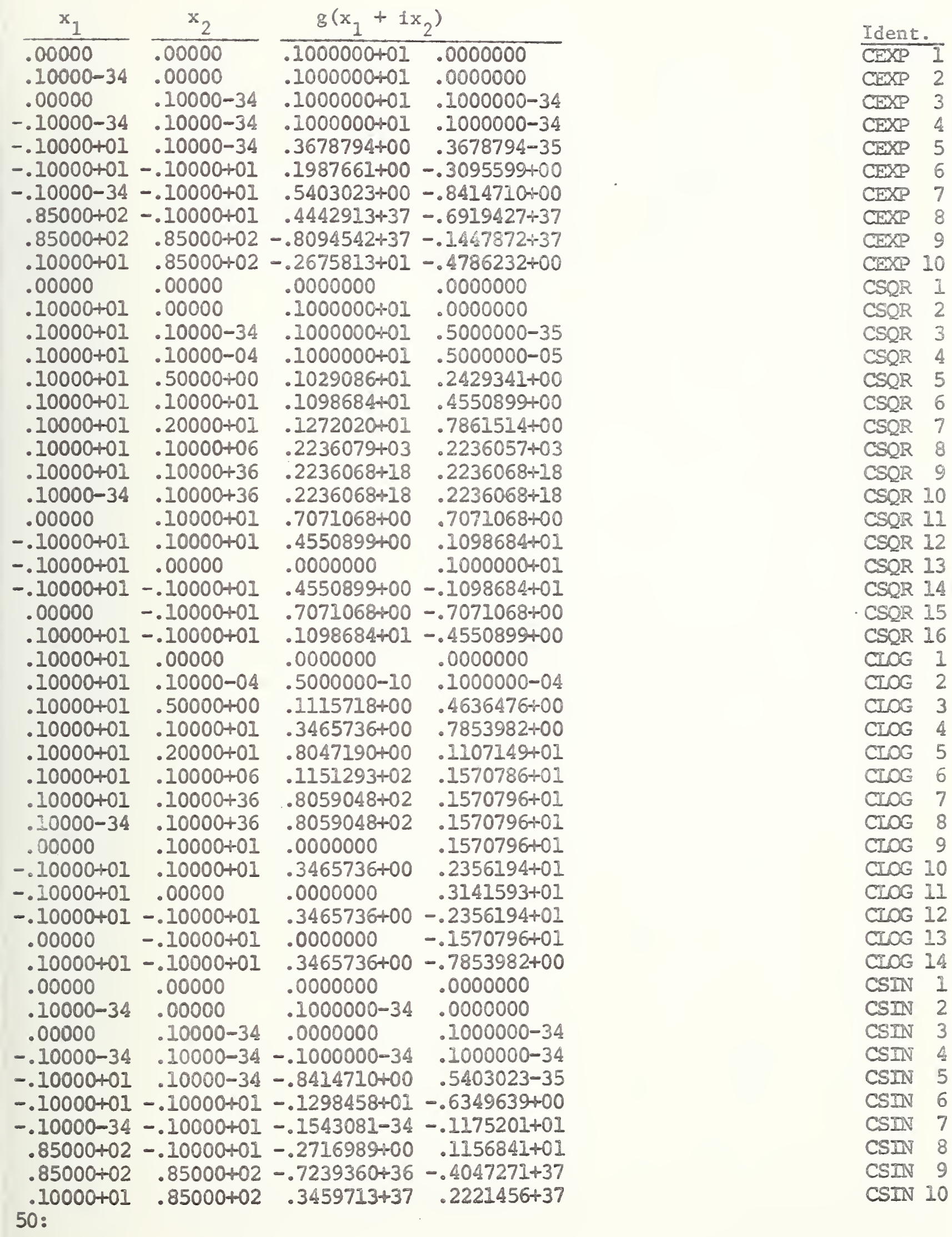

Table 3. Test data for the complex functions. 



$\begin{array}{ccccc}\frac{x_{1}}{.00000} & \frac{x_{2}}{.00000} & & \frac{g\left(x_{1}+1 x_{2}\right)}{.1000000+01} & .0000000 \\ .10000-34 & .00000 & .1000000+01 & .0000000 \\ .00000 & .10000-34 & .1000000+01 & .0000000 \\ -.10000+01 & .10000-34 & .5403023+00 & .8414710-35 \\ -.10000+01 & -.10000+01 & .8337300+00 & -.9888977+00 \\ -.10000-34 & -.10000+01 & .1543081+01 & -.1175201-34 \\ .85000+02 & -.10000+01 & -.1518973+01 & -.2069243+00 \\ .85000+02 & .85000+02 & -.4047271+37 & . .7239360+36 \\ .10000+01 & .85000+02 & .2221456+37 & -.3459713+37 \\ 59 . & & & & \end{array}$

\begin{tabular}{l} 
Ident. \\
\hline $\cos 1$ \\
$\cos 2$ \\
$\cos 3$ \\
$\cos 4$ \\
$\cos 5$ \\
$\cos 5$ \\
$\cos 7$ \\
$\cos 5$ \\
$\cos 9$
\end{tabular}

Table 3 (cont.). Test data for the complex functions. 



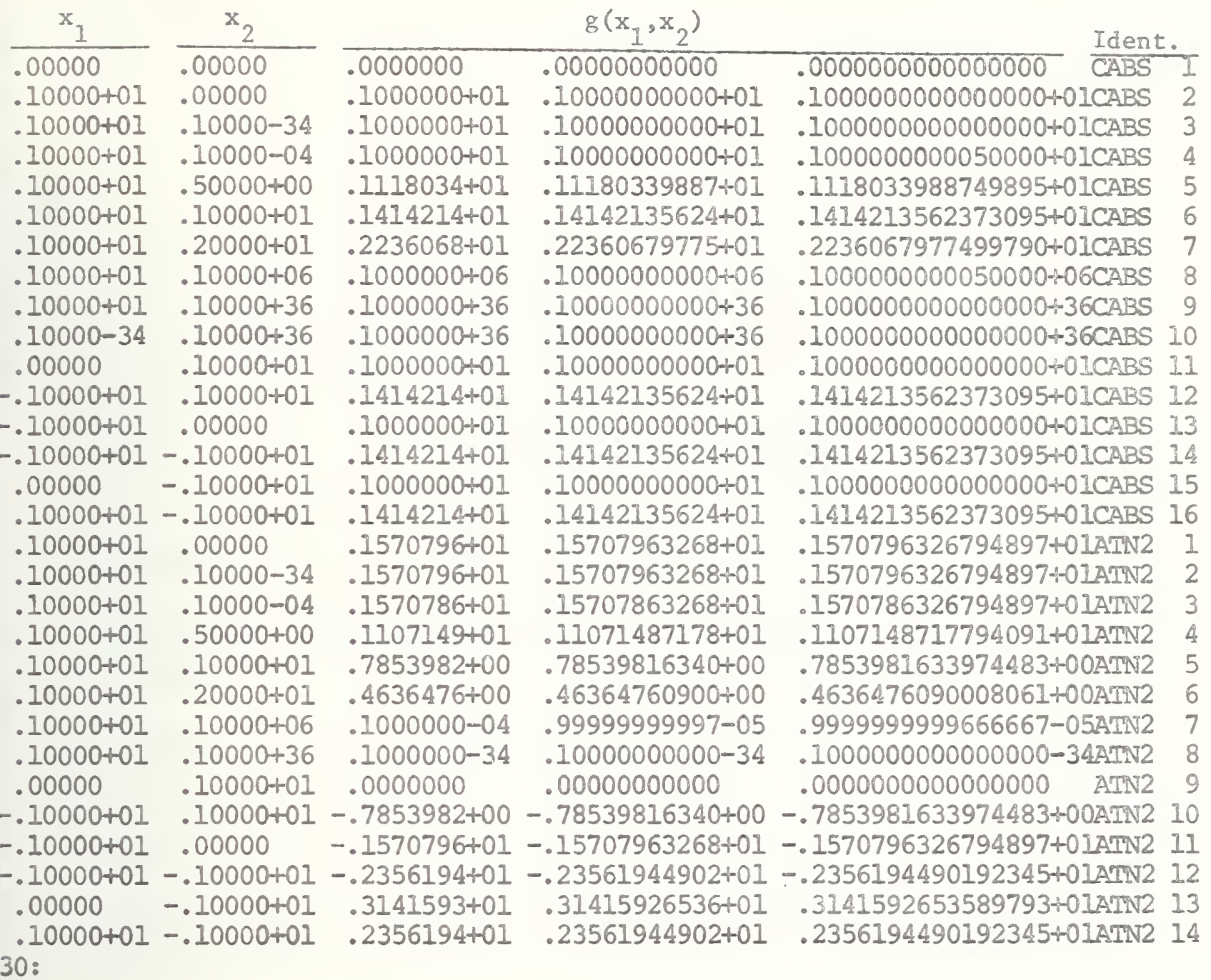

Table 4. Test data for $g\left(x_{1}, x_{2}\right)=\left|x_{1}+i x_{2}\right|$ and $g\left(x_{1}, x_{2}\right)=\arctan \left(x_{1}, x_{2}\right)$. 

4. References

1. Abramowitz, M. and Stegun, I.A., Eds. Handbook of Mathematical Functions with Formulas, Graphs, and Mathematical Tables, National Bureau of Standards, AMS 55, 1964.

2. Hart, J.F., et.al. Computer Approximations, SIAM Series in Applied Mathematics, John Wiley \& Sons, Inc., 1968.

3. Lyusternik, L.A., Chervonenkis, O.A., and YanpoI'skii, A.R., Handbook for Computing Elementary Functions, Pergamon Press, 1965.

4. Olver, F.W.J., A New Approach to Error Arithmetic, SIAM J. Numer. Analysis, 15, pp. 368-393, 1978.

5. Sterbenz, P.H., Floating-Point Computation, Prentice-Hall, Inc., 1974. 

NBS-IIAA IFE, , I, II

\begin{tabular}{|c|c|c|}
\hline $\begin{array}{l}\text { U.S. OEPT. OF COMM. } \\
\text { BIBLIOGRAPHIC DATA } \\
\text { SHEET }\end{array}$ & $\begin{array}{l}\text { 1. IIIIIC AIION OK KIPPORT NO. } \\
\text { NBSIR 78-1478 }\end{array}$ & $\begin{array}{l}\text { 2. Gov't Accession } \\
\text { No. }\end{array}$ \\
\hline
\end{tabular}

4. TI11.I: ANI) SUIBITI.I:

A Universal Set of Test Data for Computer Implementations of Elementary Mathematical Functions

7. AI:TIIOR(L)

Daniel W. Lozier

9. ['I:KFORMING;ORG;ANIZATION NAME AND ADDRESS

NATIONAL BUREAU OF STANDARDS

DEPARTMENT OF COMMERCE

WASHINGTON, D.C. 20234

12. Spunsoring Organization Name and Complete Address (Street, City, State, ZIP)

5. Publication Date

6. Performing Organizarion ( ode

3. Recipient's Accession No.

8. Performing Organ. Report No.

10. Project/Task/Work Unut No. 7110143

11. Contract/Grant No.

13. Type of Report \& Period Covered

14. Sponsoring Agency Code

16. ABSTRAC:T (A 200-word or less tactual summary of most significant information. If document includes a significant bibliography or literature survey, mention it here.)

A short table of values of 20 mathematical functions commonly found in computer libraries is given. The data was chosen to sample the functions throughout the range of a typical floating-point arithmetic. Computer implementations can be tested by comparing computed function values against the tabular values. A discussion on how to interpret the test results is included, for both decimal and non-decimal implementations.

17. KEY WORDS ( $\mathrm{s}$ ix to twelve entries; alphabetical order; capitalize only the first letter of the first key word unless a proper name; separated by semicolons)

Fortran library testing; Mathematical function testing; Elementary function testing; Fortran library test data; Test data for mathematical functions; Test data for elementary functions.

18. AVAll.AIII.TY [x Unlimited

lior (official Distribution. Do Not Release w NTIS

Order lirum Sup. of Doc., U.S. (iovernment l'rinting Office Washington, 1). (. 20)402, Si) (au. No. (13)

(Order lirom National Technical Information Service (NTIS) $x$ Springfield, Virginia 22151

\begin{tabular}{|c|c|}
\hline $\begin{array}{l}\text { 19. SECURITY CI.ASS } \\
\text { (TIIS REPURT) }\end{array}$ & 21. NO. OF PAGES \\
\hline UNCL ASSIFIED & 29 \\
\hline $\begin{array}{l}\text { 20. SECURITY ( LASS } \\
\text { (THISPAGE) } \\
\text { UNCI.ASYIIIED) }\end{array}$ & $\begin{array}{l}\text { 22. Price } \\
\$ 4.50\end{array}$ \\
\hline
\end{tabular}


6484

-. 



